\title{
Synthesis and Luminescent Properties of Europium Complexes Covalently Bonded to Hybrid Materials Based on MCM-41 and Poly(Ionic Liquids)
}

\author{
Xiaolong Zheng, Meiyu Wang and Qiuping Li * (1) \\ School of Materials and Chemical Engineering, Ningbo University of Technology, No. 201, Fenghua Road, \\ Jiangbei District, Ningbo 315211, China; Zhengxiaolong142@sina.com (X.Z.); Wangmeiyu130@163.com (M.W.) \\ * Correspondence: liqiuping@nbut.edu.cn; Tel.: +86-574-87081240
}

Received: 13 April 2018; Accepted: 24 April 2018; Published: 26 April 2018

\begin{abstract}
Due to the wide potential application in the fields of sensing, lighting materials, and optical-electrical multifunctional devices, rare earth complex hybrid materials have been studied extensively over the past decades. A poly(ionic liquid)/mesoporous-based hybrid system which has been functionalized by the covalently linked europium complexes was reported here. Through surface modification with a coupling agent bearing an vinyl group, MCM-41 was chosen as the carrier matrix for poly(ionic liquids) (PILs) and europium compounds, and based on that, novel luminescent hybrid materials were prepared by confining the ionic liquid and europium complexes into the inorganic $\mathrm{Si}-\mathrm{O}$ frameworks. The resulting organic/inorganic materials are chemically bonded hybrids which show good photoluminescent properties such as broad excitation spectra, line-like emission spectra, and long luminescence lifetimes. The PILs/MCM- $41 / \mathrm{Eu}^{3+}$ hybrid reported here is a rare earth multifunctional material which is believed to have potential applications in the field of optical-electrical materials.
\end{abstract}

Keywords: rare earth; ionic liquid; mesoporous material; hybrid material; luminescence

\section{Introduction}

Because of their intense line-like luminesce emission and long lifetime, lanthanide complexes have been found to have diverse chemical and biological applications, such as light-emitting devices, luminescent optical fibers, fluorescent probes, and so on [1-3]. However, the poor stability of traditional lanthanide complexes has still limited their practical applications in some cases. Fortunately, the inserting of lanthanide complexes into various hybrid structures might offer an effective solution [4], which could be easily accomplished by the use of tailorable organic ligands [5-7]. Thus, the luminescent lanthanide hybrid materials have attracted much attention, owing to their powerful abilities of combining the outstanding photoluminescence of lanthanide elements with various matrices [8].

The important matrices for constructing luminescent lanthanide complex hybrid materials mainly include organic polymers [9], inorganic silica [10], inorganic zeolite [11], or inorganic-organic hybrid matrices and so on [12]. Recently, poly(ionic liquids) (PILs) have been found to have huge potential applications in some aspects of the material science field [13]. Since the ionic liquid was used for synthesizing lanthanide hybrid luminescent materials [14,15], many works have been focused on the using of PILs for lanthanide hybrid materials. $\mathrm{Ru}$ [16] has studied the synthesis of terpyridine moiety-modified PILs and their usage for constructing europium-based hybrid materials. Li [17] has reported the using of a bifunctional ionic liquid which carries a benzoic acid group and which acts as a matrix and second ligand simultaneously for preparing novel lanthanide complex functionalized hybrid PILs. The optical active hybrid materials based on PILs and inorganic matrices could 
find potential applications in the fields of optical-electrical devices such as organic light-emitting devices (OLEDs), luminescent solar concentrators, and so on $[18,19]$. However, the application of PILs for constructing inorganic-organic lanthanide complex hybrid materials has rarely been reported. Taking the covalent incorporation of europium complexes into the organic/inorganic matrices, here, the synthesis of novel luminescent rare earth complex functionalized materials based on the poly(ionic liquid)/mesoporous MCM-41 hybrid matrices has been discussed. The method reported here is a practice of constructing novel optical-electrical multifunctional materials, the functional moieties of which are linked covalently. Hence, a series of class II hybrid photoactive materials has been gained, which show good thermal stability and photoluminescence properties. Moreover, the resulting europium complex hybrid materials have been measured in detail with respect to its physical properties.

\section{Materials and Methods}

The 1-allyl-3-methylimidazolium chloride (AMIMCl) ionic liquid was purchased from Shanghai Cheng Jie chemical Co. LTD, while other reagents were provided by Aladdin reagents Co. LTD (Shanghai, China). The MCM-41 material was prepared according to the general procedures, and then was refluxed with ethanol in Soxhlet extraction for two days to remove the surfactant. After that, the MCM-41 was transferred into a solution of vinyltrimethoxysilane (VTMOS) in toluene and then refluxed at $80^{\circ} \mathrm{C}$ for $12 \mathrm{~h}$. The vinyl-functionalized MCM- 41 was obtained from the solution and purified with toluene and ethanol, marked as MCM-41-Vinyl. The polymerizable europium complexes $\mathrm{Eu}(\mathrm{TTA})_{3} \mathrm{~L} 2$ (TTA = 2-thenoyltrifluoroacetone, $\mathrm{L} 2$ = acrylic acid (AA), methacrylic acid (MAA), undecylenic acid (UA)) were synthesized by the well-established protocol [20]. The hybrid luminescent materials were prepared according to the following steps: A mixture of AMIMCl (1 mmol), $\mathrm{Eu}(\mathrm{TTA})_{3} \mathrm{~L} 2(0.1 \mathrm{mmol}), \mathrm{MCM}-41$-Vinyl $(300 \mathrm{mg})$, and AIBN $(10 \mathrm{mg})$ initiator were dispersed in dry $\mathrm{N}, \mathrm{N}$-Dimethylformamide (DMF, $10 \mathrm{~mL}$ ) and sonicated for $30 \mathrm{~min}$. The homogeneous solution was then heated to $70{ }^{\circ} \mathrm{C}$ with continuous stirring for $24 \mathrm{~h}$ under nitrogen atmosphere. The resulting solid material was collected by filtration and washed with ethanol and further purified by Soxhlet extraction with boiling ethanol for $48 \mathrm{~h}$ and finally dried in vacuum condition. The resulting materials were assigned as Eu@MCM-41-P (IL-AA), Eu@MCM-41-P (IL-MAA), and Eu@MCM-41-P (IL-UA), respectively. The hybrid materials were comprehensively characterized by XRD (Bruker D8, Ningbo University of Technology, Ningbo, China), FTIR spectroscopy (FTIR-8400S, Ningbo University of Technology, Ningbo, China), SEM (JEOL2011 microscope, Ningbo University of Technology, Ningbo, China), and TGA (Netzsch TG209F, Ningbo University of Technology, Ningbo, China) measurements. The photoluminescent behavior of materials was investigated by Hitachi F-4600 (Ningbo University of Technology, Ningbo, China) and Edinburgh Instrument FLS920 spectrophotometer (Tongji University, Shanghai, China).

\section{Results and Discussion}

The synthetic routes and schematic representation of europium complex hybrid materials are given in Figure 1. The digital photographs of powders of Eu@MCM-41-P (IL-AA), Eu@MCM-41-P (IL-MAA), and Eu@MCM-41-P (IL-UA) under daylight (top) and UV lamp (bottom) are also shown, and the characteristic emission of the $\mathrm{Eu}^{3+}$ ion was exhibited when exposed to UV light. The construction of hybrid materials was investigated by FTIR spectroscopy (Figure 2). As shown in the figure, the strongest broad bands appearing at about $1075 \mathrm{~cm}^{-1}$ in all spectra were assigned to $\mathrm{Si}-\mathrm{O}-\mathrm{Si}$ stretching vibrations of the mesoporous framework. The band at $1660 \mathrm{~cm}^{-1}$ of MCM-41-Vinyl was assigned to the stretching vibration of the $\mathrm{C}=\mathrm{C}$ group, which meant that the surface modification of MCM-41 was successful. The absorption band at about $1575 \mathrm{~cm}^{-1}$ of the resulting hybrid materials (Figure $2 \mathrm{c}-\mathrm{e}$ ) were associated with the bending vibration of the imidazole ring that comes from the AMIMCl ionic liquid. In addition, the bands at $1600 \mathrm{~cm}^{-1}$ and $1415 \mathrm{~cm}^{-1}$ in the spectra of hybrid materials (Figure 2c-e) can be assigned to the asymmetric and symmetric vibrations of the 
COO group, respectively; and the bands at $1536 \mathrm{~cm}^{-1}$ can be assigned to the delocalisation of the $\pi$-electron in the chelate ring which forms between $\mathrm{Eu}^{3+}$ and the TTA molecules. These indicate that the europium complex functionalized poly(ionic liquid)/MCM-41 (PIL/MCM-41) hybrid materials had been successfully synthesized.

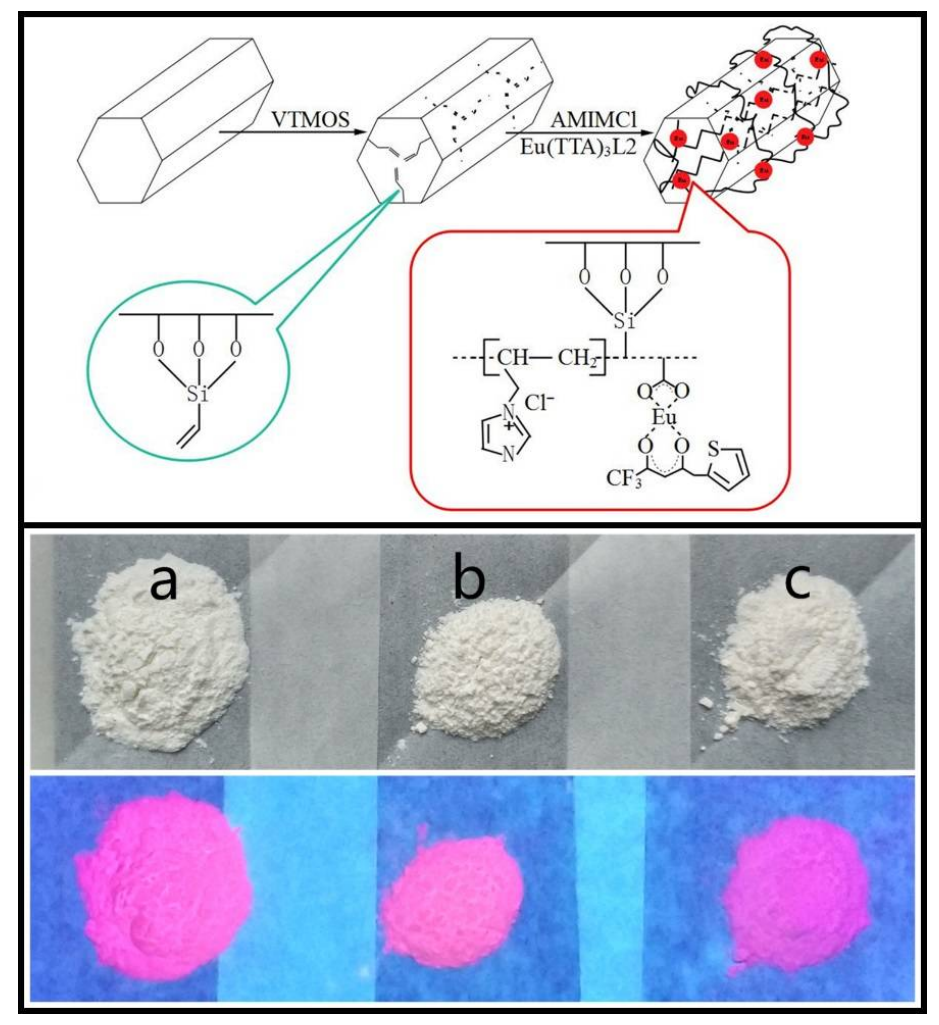

Figure 1. Synthetic scheme of the europium complex hybrid materials, and the photograph of the resulting materials under daylight (top) and 360-nm UV lamp (bottom). a = Eu@MCM-41-P (IL-AA); b= Eu@MCM-41-P (IL-MAA); c = Eu@MCM-41-P (IL-UA).

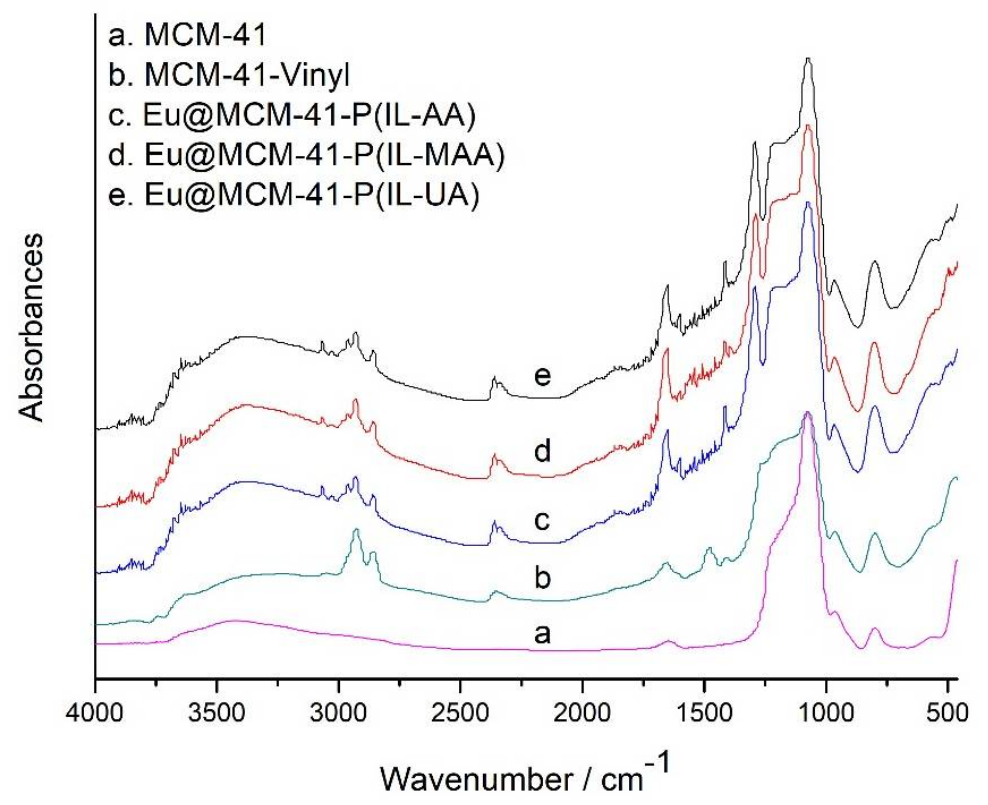

Figure 2. FTIR spectra of MCM-41, vinyl-modified MCM-41 (MCM-41-Vinyl), and the final materials. 
The mesoporous structure of the hybrid materials has also been investigated and confirmed by X-ray diffraction (Figure S1, Supplementary Materials). The transmission electron microscope images (Figure S2, Supplementary Materials) could show the flaky structure of the hybrid material, but fail to reveal the details of the microstructure of MCM-41, since its surface was covered by the poly(ionic liquid). The thermal stability of the Eu@MCM-41-P (IL-AA) hybrid material was investigated and given as the representative. As shown in Figure 3, the thermogravimetric (TG) and the corresponding derivative weight loss (DTG) analysis revealed that the PIL/MCM-41-based hybrid materials showed a two-step weight loss approach, which was associated with the decomposition of polymer compositions attaching onto the exterior of MCM-41 and that of the one confined into the tunnel of MCM-41. Compared to the general decomposition temperatures of polymers, the hybrid materials possess a wider decomposition temperature interval, which can be seen as a sign of the improvement of the thermal stability.

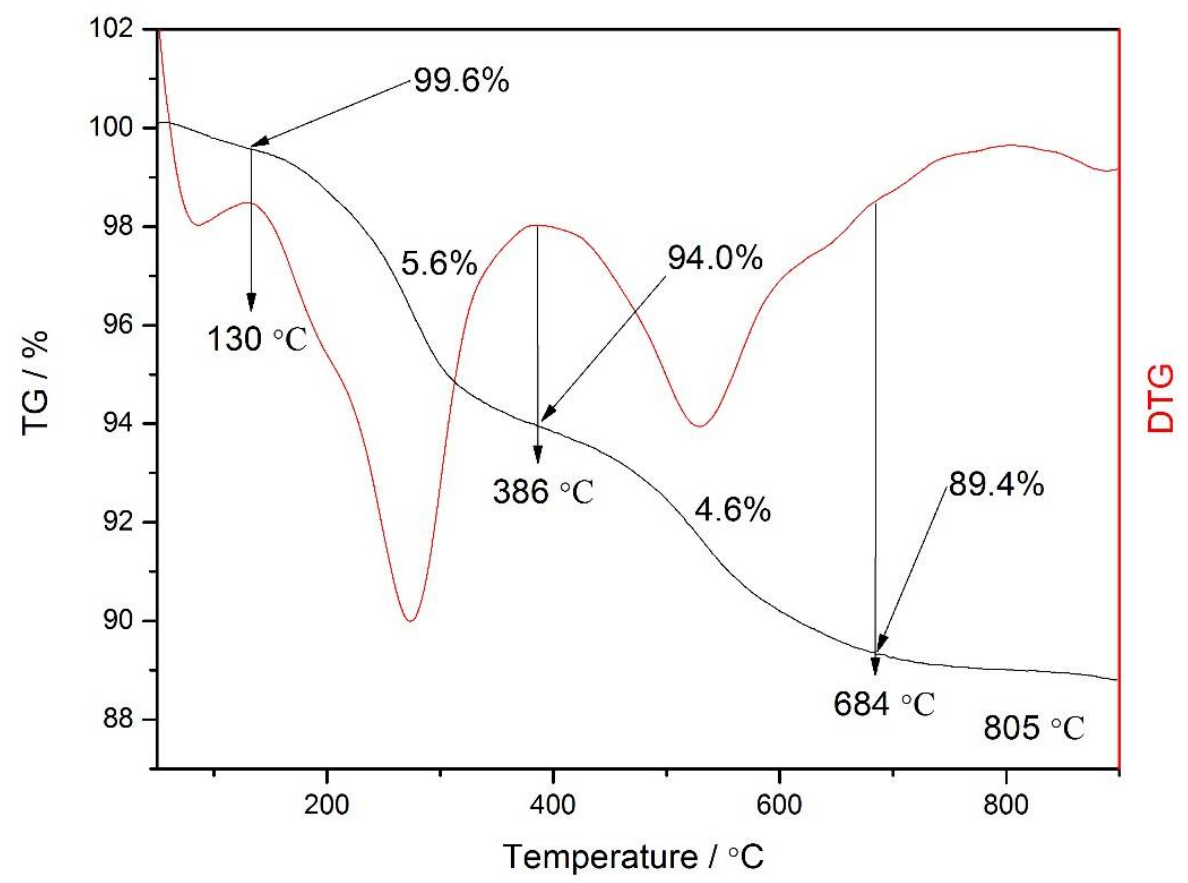

Figure 3. Thermogravimetric TG and derivative weight loss (DTG) curves of Eu@MCM-41-P (IL-AA).

The photoluminescent properties of the obtained hybrid materials have been investigated under room temperature. As shown in Figure 4A, the excitation spectra of Eu@MCM-41-P (IL-AA), Eu@MCM-41-P (IL-MAA), and Eu@MCM-41-P (IL-UA) were obtained by monitoring the characteristic emission ${ }^{5} \mathrm{D}_{0} \rightarrow{ }^{7} \mathrm{~F}_{2}$ of the $\mathrm{Eu}^{3+}$ ion at $612 \mathrm{~nm}$. It is clear from the spectra that all the hybrid materials have a broad band absorption in the range of $250-400 \mathrm{~nm}$, which is ascribed to the absorption of the TTA ligand. The maximum peaks for hybrids center at about $360 \mathrm{~nm}$, which is selected as the fixed excitation wavelength for measuring the emission spectra and luminescent lifetimes. As shown in Figure 4B, the emission spectra of all hybrid materials gave fine lines around 580, 593, 612, 653, and $703 \mathrm{~nm}$, which could be assigned to the ${ }^{5} \mathrm{D}_{0} \rightarrow{ }^{7} \mathrm{~F}_{\mathrm{J}}(J=0-4)$ transitions of the Eu ${ }^{3+}$ ion. All the emission spectrums were dominated by the very intense ${ }^{5} \mathrm{D}_{0} \rightarrow{ }^{7} \mathrm{~F}_{2}$ transition line, which is responsible for the red luminescence emission of the hybrid materials. 


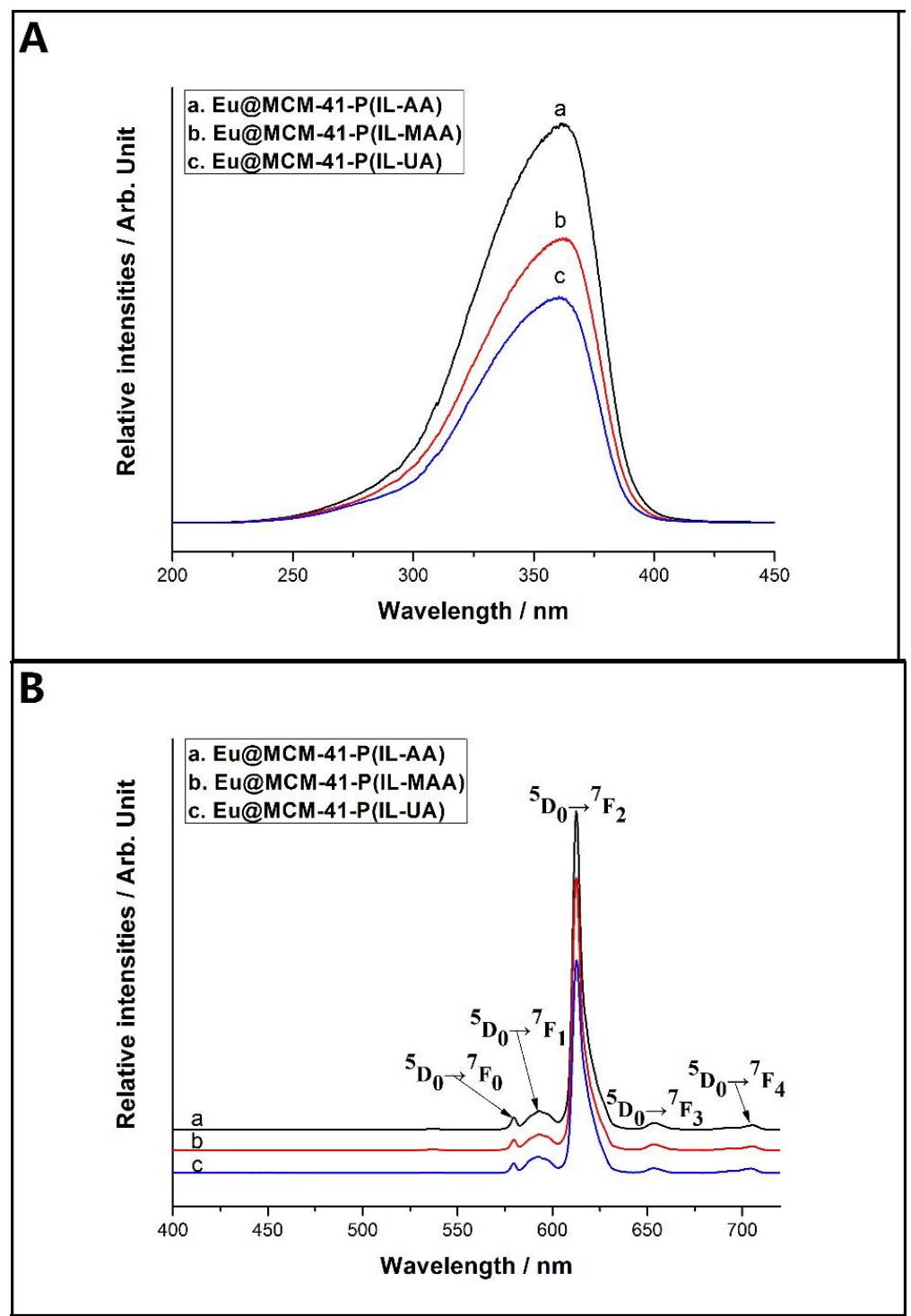

Figure 4. Excitation ((A) monitored at $\lambda=612 \mathrm{~nm})$ and emission $((\mathbf{B})$ excited with $\lambda=360 \mathrm{~nm})$ spectra of the hybrid materials.

The luminescence decay properties of the hybrid materials shown in Figure 5 fitted well to a single exponential function. The decay times were calculated to be 399.40, 397.41, and $321.48 \mu \mathrm{s}$ for Eu@MCM-41-P (IL-AA), Eu@MCM-41-P (IL-MAA), and Eu@MCM-41-P (IL-UA), respectively. Furthermore, based on the emission spectra and the lifetime $(\tau)$, the ${ }^{5} \mathrm{D}_{0}$ quantum efficiencies of all the europium complex hybrid materials were estimated according to the Juud-Ofelt theory [21]. The detailed methods are listed below:

$$
\begin{gathered}
A_{0 J}=A_{01} \times \frac{I_{0 J}}{I_{01}} \times \frac{v_{01}}{v_{0 J}} \\
A_{r}=\sum A_{0 J}=A_{00}+A_{01}+A_{02}+A_{03}+A_{04} \\
\frac{1}{\tau}=A_{r}+A_{n r} \\
\eta=\frac{A_{r}}{A_{r}+A_{n r}}
\end{gathered}
$$




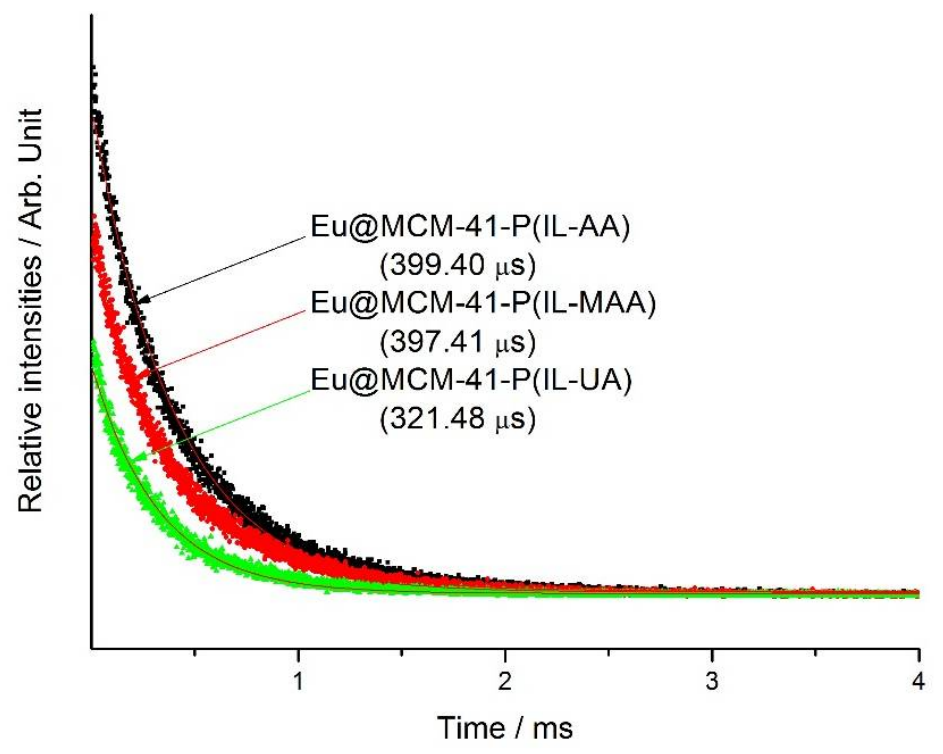

Figure 5. Luminescence decay curve for the ${ }^{5} \mathrm{D}_{0} \rightarrow{ }^{7} \mathrm{~F}_{2}$ transition $(612 \mathrm{~nm})$ of the hybrid materials.

Here, $A_{01}$ is Einstein's coefficient of spontaneous emission between ${ }^{5} \mathrm{D}_{0}$ and ${ }^{7} \mathrm{~F}_{1}$, the value of which is theoretically determined to be $50 \mathrm{~s}^{-1} ; I_{0 J}$ is the ${ }^{5} \mathrm{D}_{0} \rightarrow{ }^{7} \mathrm{~F}_{J}(J=0 \sim 4)$ transition intensities calculated from the peaks; and the $v_{0 J}(J=0 \sim 4)$ is the energy barycenter of the corresponding emission peaks. Moreover, the $A_{r}$ is the rate of radiative transition, while $A_{n r}$ is that of nonradiative transition. Based on these, the luminescence quantum efficiencies of europium complex hybrid materials can be seen as equal to the ratio of $A_{r}$ to the value of $\left(A_{r}+A_{n r}\right)$. The obtained luminescence data are summarized in Table 1 . All the luminescent quantum efficiencies of the hybrid materials are over thirty percent, as shown in the table, and it is clear that Eu@MCM-41-P (IL-AA) (37.1\%) and Eu@MCM-41-P (IL-MAA) (36.1\%) have similar values, higher than that of Eu@MCM-41-P (IL-UA) (33.2\%), which is consistent with the result of luminescence lifetimes. From these results, it is reasonable to assume that the second ligand (i.e., UA) with a long chain structure works against the photoluminescent properties. On the other hand, the reason why the hybrid materials Eu@MCM-41-P (IL-AA) and Eu@MCM-41-P (IL-MAA) show very similar luminescence quantum efficiencies and lifetimes is probably due to having similar molecular structures to those of AA and MAA ligands.

Table 1. Luminescent data for the hybrid materials.

\begin{tabular}{ccccc}
\hline & $\boldsymbol{\tau} \mathbf{( \mu \mathbf { s } ) ^ { \mathbf { a } }}$ & $\boldsymbol{A}_{\boldsymbol{r}}\left(\mathbf{s}^{-\mathbf{1}}\right)$ & $\boldsymbol{A}_{\boldsymbol{n} \boldsymbol{r}}\left(\mathbf{s}^{-\mathbf{1}}\right)$ & $\boldsymbol{\eta} \mathbf{( \% )} \mathbf{b}^{\mathbf{b}}$ \\
\hline Eu@MCM-41-P (IL-AA) & 399.4 & 928.0 & 1573.3 & 37.1 \\
Eu@MCM-41-P (IL-MAA) & 397.4 & 907.1 & 1605.6 & 36.1 \\
Eu@MCM-41-P (IL-UA) & 321.5 & 1031.7 & 2075.8 & 33.2 \\
\hline
\end{tabular}

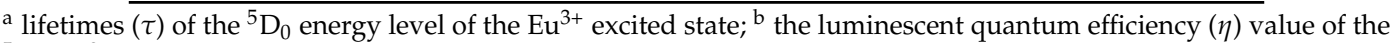
${ }^{5} \mathrm{D}_{0} \mathrm{Eu}^{3+}$ excited state calculated from decay times and emission intensities.

\section{Conclusions}

In summary, a series of europium-based hybrid materials Eu@MCM-41-P (IL-L2) (L2 = AA, MAA, UA) was prepared successfully through an in situ polymerization of polymerizable europium complexes, polymerizable ionic liquids, and vinyl-modified MCM-41. The resulting luminescent materials are chemically bonded organic/inorganic hybrids that could be excited by $360-\mathrm{nm}$ light effectively and exhibited strong red emission mainly originating from the ${ }^{5} \mathrm{D}_{0} \rightarrow{ }^{7} \mathrm{~F}_{2}$ transition $(612 \mathrm{~nm})$ of $\mathrm{Eu}^{3+}$ ions. The materials have good thermal stability and outstanding photoluminescent properties. The luminescent quantum efficiencies of Eu@MCM-41-P (IL-AA) and Eu@MCM-41-P (IL-MAA) are 
very close and higher than that of Eu@MCM-41-P (IL-UA), apparently indicating that a smaller second ligand would beneficial for the photoluminescent properties of the europium complex hybrid materials reported here. The present investigation manifested that the PILs/MCM-41 hybrid is a good matrix for constructing novel rare earth hybrid materials. Future works will focus on its application in optical/electrical fields.

Supplementary Materials: The following are available online at http:/ / www.mdpi.com/1996-1944/11/5/677/s1: Figure S1: XRD patterns of MCM-41 and the final materials; Figure S2: TEM of the Eu@MCM-41-P (IL-AA).

Author Contributions: Xiaolong Zheng and Meiyu Wang performed the experiments; Qiuping Li conceived and designed the experiments, analyzed the data, and wrote the paper.

Funding: This research was funded by the Natural Science Foundation of Zhejiang Province (LQ14B010001) and the Natural Science Foundation of Ningbo (2016A610105).

Conflicts of Interest: The authors declare no conflict of interest regarding the publication of this paper.

\section{References}

1. Han, B.; Dai, Y.; Zhang, J.; Shi, H. Luminescence properties of a novel yellow-emitting phosphor $\mathrm{NaLaMgWO}_{6}: \mathrm{Dy}^{3+}$. Mater. Lett. 2017, 204, 145-148. [CrossRef]

2. Liao, X.; Jiang, X.; Yang, Q.; Wang, L.; Chen, D. Spectral Properties of $\mathrm{Er}^{3+} / \mathrm{Tm}^{3+}$ Co-Doped ZBLAN Glasses and Fibers. Materials 2017, 10, 486. [CrossRef] [PubMed]

3. Lönnrot, M.; Sjöroos, M.; Salminen, K.; Maaronen, M.; Hyypiä, T.; Hyöty, H. Diagnosis of enterovirus and rhinovirus infections by RT-PCR and time-resolved fluorometry with lanthanide chelate labeled probes. J. Med. Virol. 2016, 59, 378-384. [CrossRef]

4. Binnemans, K. Lanthanide-Based Luminescent Hybrid Materials. Chem. Rev. 2009, 109, 4283-4374. [CrossRef] [PubMed]

5. Caricato, M.; Coluccini, C.; Griend, D.A.V.; Forni, A.; Pasini, D. From red to blue shift: Switching the binding affinity from the acceptor to the donor end by increasing the $\pi$-bridge in push-pull chromophores with coordinative ends. New J. Chem. 2013, 37, 2792-2799. [CrossRef]

6. $\quad$ Aguiar, F.P.; Costa, I.F.; Espínola, J.G.P.; Faustino, W.M.; Moura, J.L.; Brito, H.F.; Paolini, T.B.; Felinto, M.C.F.; Teotonio, E.E. Luminescent hybrid materials functionalized with lanthanide ethylenodiaminotetraacetate complexes containing $\beta$-diketonate as antenna ligands. J. Lumin. 2016, 170, 538-546. [CrossRef]

7. Pacini, A.; Caricato, M.; Ferrari, S.; Capsoni, D.; Antxon, M.D.I.; Muñoz-Gerra, S.; Pasini, D. Poly $(\gamma$-glutamic acid) esters with reactive functional groups suitable for orthogonal conjugation strategies. J. Polym. Sci. Part A Polym. Chem. 2015, 50, 4790-4799. [CrossRef]

8. Yan, B. Recent Progress in Photofunctional Lanthanide Hybrid Materials. RSC Adv. 2012, 2, 9304-9324. [CrossRef]

9. Machado, K.; Mukhopadhyay, S.; Videira, R.A.; Mishra, J.; Mobin, S.M.; Mishra, G.S. Polymer encapsulated scorpionate $\mathrm{Eu}^{3+}$ complexes as novel hybrid materials for high performance luminescence applications. RSC Adv. 2015, 5, 35675-35682. [CrossRef]

10. Li, W.X.; Zheng, Y.S.; Cao, X.F.; Bai, J.; Fu, Z.F.; Bao, J.R.; Li, Y.L. Preparation, characterization, and luminescence properties of dysprosium perchlorate with MABA-Si and phen or dipy complexes as well as $\mathrm{SiO}_{2} @ \mathrm{Dy}(\mathrm{MABA}-\mathrm{Si})$ core-shell structure nanometermeter luminescent composites. J. Lumin. 2016, 178, 470-478. [CrossRef]

11. Wang, Y.; Li, H.; Zhang, W.; Liu, B. Luminescence properties of nanozeolite L grafted with terbium organic complex. Mater. Lett. 2008, 62, 3167-3170. [CrossRef]

12. Feng, J.; Zhang, H. Hybrid materials based on lanthanide organic complexes: A review. Chem. Soc. Rev. 2013, 42, 387-410. [CrossRef] [PubMed]

13. Gao, M. R.; Yuan, J.; Antonietti, M. Ionic liquids and poly(ionic liquid)s for morphosynthesis of inorganic materials. Chemistry 2016, 23, 5391-5403. [CrossRef] [PubMed]

14. Lunstroot, K.; Driesen, K.; Nockemann, P. Luminescent Ionogels Based on Europium-Doped Ionic Liquids Confined within Silica-Derived Networks. Chem. Mater. 2006, 18, 5711-5715. [CrossRef]

15. Zhou, F.; Wang, T.; Li, Z.; Wang, Y. Transparent and luminescent ionogels composed of $\mathrm{Eu}^{3+}$ coordinated ionic liquids and poly(methyl methacrylate). Luminescence 2015, 30, 1303-1307. [CrossRef] [PubMed] 
16. Li, Z.; Wang, J.; Chen, M.; Wang, Y. Lanthanide Luminescence Improvement by Using a Functional Poly(Ionic Liquid) as Matrix and Co-ligand. Chem. Asian J. 2016, 11, 745-749. [CrossRef] [PubMed]

17. Ru, Q.; Xue, Z.; Wang, Y.; Liu, Y.; Li, H. Luminescent Materials of Europium(III) Coordinated by a Terpyridine-Functionalized Poly(Ionic Liquid). Eur. J. Inorg. Chem. 2013, 2014, 469-474. [CrossRef]

18. Wang, H.F.; Wang, Y.G.; Zhang, L.; Li, H.R. Transparent and luminescent ionogels based on lanthanide-containing ionic liquids and poly(methyl methacrylate) prepared through an environmentally friendly method. RSC Adv. 2013, 3, 8535-8540. [CrossRef]

19. Yuan, J.Y.; Antonietti, M. Poly(ionic liquid)s: Polymers expanding classical property profiles. Polymer 2011, 52, 1469-1482. [CrossRef]

20. Melby, L.R.; Rose, N.J.; Abramson, E.; Caris, J.C. Synthesis and Fluorescence of Some Trivalent Lanthanide Complexes. J. Am. Chem. Soc. 1964, 86, 5117-5125. [CrossRef]

21. Hehlen, M.P.; Brik, M.G.; Krämer, K.W. 50th anniversary of the Judd-Ofelt theory: An experimentalist's view of the formalism and its application. J. Lumin. 2013, 136, 221-239. [CrossRef]

(C) 2018 by the authors. Licensee MDPI, Basel, Switzerland. This article is an open access article distributed under the terms and conditions of the Creative Commons Attribution (CC BY) license (http:/ / creativecommons.org/licenses/by/4.0/). 